\title{
ARTICLE
}

\section{An Automatic Image Analysis Methodology for the Measurement of Droplet Size Distributions in Liquid-Liquid Dispersion: Round Object Detection}

\author{
K. Gawryszewski ${ }^{a}$, Z. A. Rana ${ }^{\mathrm{a}}$ K. W. Jenkins ${ }^{\mathrm{a}}$, P. Ioannou ${ }^{\mathrm{b}}$ and D. Okonkwo ${ }^{\mathrm{b}}$ \\ ${ }^{a}$ Centre for Computational Engineering Sciences, Cranfield University, Cranfield, \\ Bedfordshire, UK; ${ }^{\mathrm{b}}$ BHR Group, The Fluid Engineering Centre, Cranfield, Bedfordshire, UK
}

\author{
ARTICLE HISTORY \\ Compiled November 8, 2018
}

\begin{abstract}
This article presents an efficient and economical automatic image analysis technique for the droplet characterization in a liquid-liquid dispersion. The methodology employs a combination of the Satoshi Suzuki's (Suzuki, 1985) find contours algorithm and the method of minimal enclosing circle identification, proposed by Emo Welzl (Welzl, 1991), to achieve the objectives. The round object detection algorithm has been designed for the identification and verification of correct droplets in the mixture which helped to increase the accuracy of automatic detection. Tests have been performed on various sets of images obtained during several emulsification processes and contain examples of droplets which differ in size, density, volume and appearance etc. An effective communication between the two methodologies and newly introduced algorithms demonstrated an accuracy of $90 \%$ or above in the measurement of droplet size distribution and Sauter mean diameters through an automatic vision based system.
\end{abstract}

\section{KEYWORDS}

Round Object Detection; Droplet Detection; Droplet Size Distribution; Liquid-Liquid Dispersion; Image Analysis; Computer Vision.

\section{Introduction}

In chemical and process industry, the mixing process manipulates a heterogeneous system and aims to make it a more homogeneous. Such processes include pumping of water into a swimming pool to make the temperature more homogenized, mixing of two different paints or chemicals and mixing of gases in a liquid medium. Applications can be various including (but not limited to) food industry, chemical reactors, cosmetic industry, bio-fuel and many more. A consortium consisting of various industrial sectors run by our industrial collaborator, referred to as the Fluid Mixing Processes (FMP), looks at the research of single or multi-phase mixing processes in various applications. One of the areas of importance for FMP is the the dispersion of two immiscible liquids, normally referred to as liquid-liquid dispersion (LLD). In LLD two liquids phases, such as oil and a water-based liquid, would segregate when poured in to a container without mixing, however if correctly agitated using an impeller they would form dispersion. 
When the two liquid phases are dispersed, they form an emulsion which contains droplets of the dispersed phase in the continuous phase that do not coalesce.

Droplet characterization, specifically measurement of the droplet size distribution (DSD), is one of the most important parameters in LLD as it affects mass transfer rates and other physical properties of the emulsion. Various factors can influence this such as the concentration of the dispersed phase, viscosity and the mixing process characteristics (e.g. size and type of impeller and rotational speed). FMP has developed a number of correlations to predict the average equilibrium droplet diameter for a given set of process conditions, however, they continuously collect data to improve the range of applicability. In many cases surfactants are used, which are chemical additives to assist the dispersion by lowering the interfacial tension and stabilize the system against coalescing (referred to as a surfactant-stabilized or non-coalescing system).

Over the years, several efforts have been put in to accurately measuring the DSD in an LLD, however this particular area is still of major interest to the industry and researchers. Historically a manual approach based upon external sample analysis was used for the measurement of DSD and Sauter mean diameter in the industry, however, in-situ DSD measurements have been found to be challenging (Maaß, Rojahn, Hnsch, \& Kraume, 2012). Although there is significant research (Narsimhan \& Goel, 2001; Tcholakova, Denkov, \& Danner, 2004; Wright \& Ramkrishna, 1994) carried out in the area of external sample analysis, there are several limitations to this methodology. The group of in-situ/on-line methods (which include techniques such as acoustic, laser diffraction, light back scattering, videos and endoscopes) is dedicated to studies which require knowledge of how emulsion changes its parameters throughout the mixing process (Basaran \& McClements, 1999; Chatzi, Boutris, \& Kiparissides, 1991; Niknafs, Spyropoulos, \& Norton, 2011; Richter, Voigt, \& Ripperger, 2007; G. Zhou \& Kresta, 1998). Several in-situ/on-line techniques have been proposed over the last few years (including the use of image analysis) and it has to be acknowledged that all techniques offer some advantages and disadvantages. In approaches using a stream of light, for example, a transparent system is required and as a result often are unable to make correct measurements for emulsions with high volume fraction of dispersed phase, however, this problem can be overcome by approaches using ultrasounds. The pros and cons associated with other techniques indicate that the digital imaging technique is still one of the better options and needs to be explored further (Alban, Sajjadi, \& Yianneskis, 2004; Kamp, Hnsch, Kendzierski, Kraume, \& Hellwich, 2016; Maa $\beta$, Wollny, Voigt, \& Kraume, 2011; Pacek, Moore, Nienow, \& Calabrese, 1994; Ritter \& Kraume, 2000).

Use of imaging in emulsification has been employed for several years; historically, analogue photographs have been used (Chen \& Middleman, 1967; Coulaloglou \& Tavlarides, 1976) which could make imaging one of the first and most popular techniques used for the DSD measurements. Analysis of the digital images technique can be employed both for off-line and on-line measurement of droplets with limitation for systems where high rate of droplet coalescence occurs (Alban et al., 2004). Off-line measurement method is suitable for emulsions only if the stability of the droplets is on a level warranting reliable size measurement. However, very often this stability condition is unsatisfied and additional actions are required to improve kinetic stability. Usually, a surface-active surfactant is used where in most of the cases only a small amount of it added into studied emulsion can sufficiently increase its stability and thus the reliability of the DSD analysis (Arai, Konno, Matunaga, \& Saito, 1977). The extra surfactant inside emulsion is not the only one known solution used for droplet stability improvement; diluting the collected dispersion samples with an aqueous ver- 
sion of a surfactant (Lagisetty, Das, \& Kumar, 1986; Tobin, Muralidhar, Wright, \& Ramkrishna, 1986) before size measurements, can also give desired stability.

It was noted (Pacek et al., 1994) that every interference in extracted samples from emulsion changed its properties and made measurement ineffective; especially for systems where fast coalescence and reaction exists. Such situation is not good even for short time of sampling (often less than one second) because changes in coalescence and flow of the emulsion result in significant measurement discrepancies. In (Pacek et al., 1994) proposed approach which solved this problem was suggested where they used a digital video camera to record the mixing process. After this, they manually analyzed the captured video frame by frame and thus they were able to get relevant information for characterizing dispersion of chlorobenzene in water containing various amounts of glycerol. Nowadays this method is widely used for image acquisition either for basic, relatively simple systems or complex multi phase dispersions (Galindo et al., 2005). However, parallel extraction of information from in-situ images remains a challenging task for several reasons which are pointed in (Y. Zhou, Srinivasan, \& Lakshminarayanan, 2009).

Ribeiro et al. (Ribeiro, Guimares, Madureira, \& Pinto, 2004) also utilized videos for the image acquisition process. The video was analyzed frame by frame to characterize the droplets in the studied emulsion. The manual methodology was time-consuming and implied high labor costs thus could present high rate of errors. The overview of the analysis process has been presented in (Junker, 2006) which demonstrates the use of images for droplet detection. Most of the available studies consider off-line than online sampling however, this trend is changing and these days state of the art approach is the development of on-line computer applications capable of reliable automatic, accurate and (equally important) fast analysis of acquired images. The analysis time factor is determined by two parameters namely data acquisition time (DAT) and measurement acquisition time (MAT).The DAT delivers information about the time needed to capture an image of the studied emulsion, the MAT factor is a combination of DAT and the real measurement time needed to extract relevant information from an image. Ideally, MAT should be equal to DAT (Crawley \& Malcolmson, 2004). Image analysis can not change DAT because this factor depends only on equipment specification used for emulsions photography, while MAT depends mainly on image analysis algorithms. It is obvious that the condition of minimization of the difference between MAT and DAT can never be satisfied by manual evaluation of images due to its nature. On the other hand, the automatic droplets detection gives hope as it can cope with the detection of the droplets but can also do this with as short time as possible depending upon the algorithm.

Associated with these, another challenge is the quality of the images acquired during the process which can present drawbacks such as overlapping droplets, lighting and noise. Research in this area indicates that the quality of acquired images have a big impact on the success of the automated detection of the objects of interest and their measurements (Yan, Sayad, \& Balke, 2009). The influence of the image quality of automatic measurements precision and droplets detection success was tested and presented in (Bras, Gomes, Ribeiro, \& Guimares, 2009) where the authors proposed algorithm for automatic image analysis for the measurement of DSD in an emulsion. To evaluate the robustness of this methodology against variations in image quality as objectively as possible they chose images with two different levels of quality. Firstly droplets were identified manually by authors which indicated that there were more than 100 droplets with varying parameters (such as radii, overlapping, border quality, etc.) in each image. Automatic detection was then applied and results were compared 
to each other. For better visualization of the differences, they defined two parameters namely the recall and the precision. The recall measured the proportion of droplets that the program was able to identify correctly, whereas the precision was the proportion of droplets identified by the program that are truly correct. As a result, they obtained 0.71 recall and 0.89 precision for the first image and 0.55 recall and 0.87 precision for the second image with lower quality.

Generally, all algorithms work well for designed systems but for only low coalescence - otherwise cannot give satisfying results in terms of droplets detection (Maa $\beta$ et al., 2012). However, promising results in (Alban et al., 2004; Bras et al., 2009) show a way forward. Alban et al. (Alban et al., 2004) proposed an interesting combination of image processing algorithm for arc and circle center estimation and further a pattern matching algorithm for verification of results. In this method the biggest problem was with small droplets especially when their contours are weakly visible. On the other hand, Bras et al. (Bras et al., 2009) presented an image processing algorithm which allows extracting relevant information from an image and then used well known Hough transform for detection of circles (Cohen \& Toussaint, 1977). This approach also has the biggest problem with the detection of small droplets but in both cases, it does not have a big impact on Sauter mean diameter because this diameter mainly depends on droplets whose sizes are medium to large. Then the wrong detection of small droplets cannot significantly affect the final result. The much worse drawback is high computational time. Both, the pattern matching by Alban and Hough transform by Bras are demanding in terms of complexity of calculations. It led to high MAT factor value and makes these methods less attractive for on-line measurements.

In summary, the industry requires an efficient and accurate solution for automatic droplets detection and based upon previous efforts and experience it can be deduced that a solution based on image analysis approaches can be a better candidate. This article presents a method/algorithm for automatic droplets detection and size measurement which uses image processing and images analysis techniques with the aim to cope with various kinds of dispersions either with a high or a low density of the dispersed phase. The secondary goal is to design a fast algorithm in order to satisfy on-line measurement requirements. Section 2 below represents the methodology/algorithm and Section 3 presents results on an acquired image.

\section{Automatic Droplet Detection Algorithm}

Automatic droplets detection algorithm is presented in four steps where each step involves few actions. Figure 1 shows the flow of these actions and connections with one another. In this section every step is discussed individually, however, it must be mentioned that many existing image processing techniques have found application in this project. On the other hand for image analysis, a new approach has been presented for effective and economical measurement of DSD, although using some known functions. As such, the proposed program is a combination of known methodologies and known functions which are brought together for automatic droplets detection and some of new actions forced by the new approach of image analysis.

\subsection{Parameter Adjustment}

A lot of effort has been devoted to find solution to allows for an automatic initial parametric adjustments of samples with different properties however none cope with this 


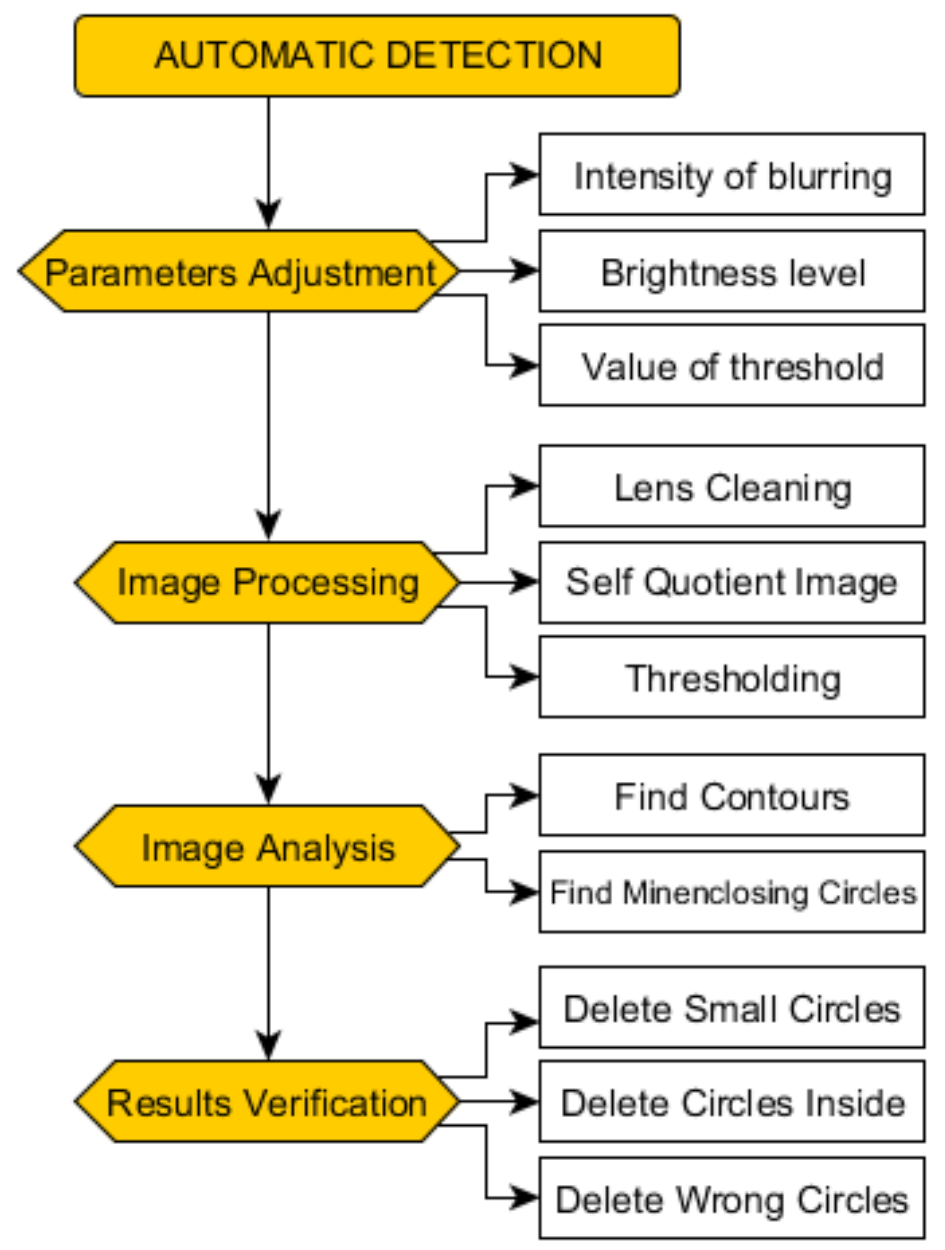

Figure 1. Schematic representation of the automatic droplets detection algorithm. 


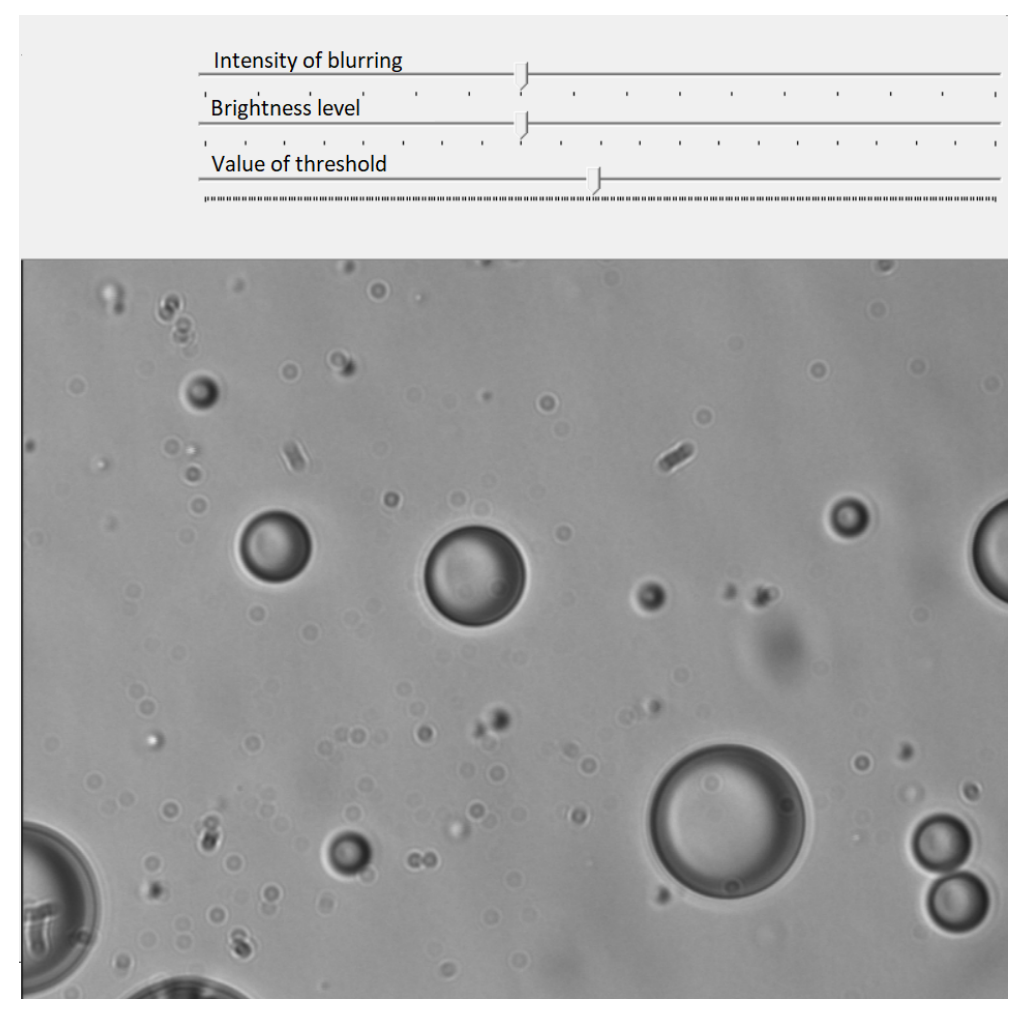

Figure 2. GUI for the parameter adjustments.

problem satisfactorily. Due to this reason the program still requires some adjustment before the automatic detection. The aim of this adjustment is to establish images suitable for analysis as all of the images are characterized by different features like droplet density, average gray level etc. Therefore, for convenience, the dynamic adjustment of the parameters has been offered. It means that an image in the studied batch can have its individual parameters however during tests first two or three images were enough for establishing parameters suitable for the rest of the images in batch. Three dynamic parameter adjustment tools provide the user a simple interface as shown in Figure 2. The decision-making regarding which parameters should be available for the user has been made after several tests on data and will be explained later in further sections below:

- Intensity of blurring

- Brightness level

- Value of threshold

\subsection{Image Processing}

Image processing is responsible for extracting and enhancing relevant information present in the image. This is precursor for the image analysis part where only the most important information in the image needs to be considered. Some known functions for edge detection and their enhancement has been tested, however techniques such as Canny, Sobel and Marr-Hildreth (Schalkoff, 1989) presented unsatisfying results; all of them caused information loss which has consequences for later processes. 
The main drawback was partial loss of contour intensity for droplets with thin borders. Additionally, some of the information in given image data was wrong and could cause errors during image analysis. For this problem, another method was required to eliminate all irrelevant information such as shadows and imperfections captured during image acquisition. Conventionally, simple contrast manipulation has been applied however, together with elimination of unnecessary data some important features has been removed as well. Overall this stage faced two problems; firstly removal of the irrelevant information without damaging important features and secondly the enhancement of important information at the same time. In this stage these challenges have been solved independently as in (Maa $\beta$ et al., 2012) which applied two steps, where in the first step redundant information is removed and in second step removal of all illumination distortions is applied.

\subsubsection{Lens cleaning}

During image acquisition, not only shapes of droplets dispersed in the emulsion are captured but some extra shapes and illumination patterns can also be observed in the images such as marks on the lenses. This type of noise is individual for every batch of acquired images and depends on lighting conditions, emulsion properties, and photography equipment. However these are mostly common to every single image in the batch and create redundant information which can be manipulated. This step is called Lens Cleaning mainly due to the such noise having its origins in dirt or particles on the lens. In order to separate these imperfections, the cumulative pixel by pixel average image is created after analyzing every image in the batch, using the Equation 1 (Maa $\beta$ et al., 2012):

$$
S=\frac{1}{\eta_{B}} \sum_{i=1}^{\eta_{B}} B_{i}
$$

The results of this process are represented in the Figure $3 \mathrm{~b}$ and has been created by the analysis of 152 images. The number of images can be crucial for this approach and the main rule is, the more images the more accurate the method. In the algorithm, a lower threshold of images count has been applied as 30; for a number of images smaller than 30 this action is omitted because it may cause the wrong separation and instead of increasing quality leads to its decrease (based on experience). Here arithmetic mean image $S$ is obtained, calculation of the new clean image is possible by subtraction of the original image and its mean image $S$ as represented by Equation 2 (Maa $\beta$ et al., 2012):

$$
B_{i}^{\prime}=B_{i}-S+\bar{S}
$$

The $\bar{S}$ is an average gray level of the $S$ image and addition of this factor is necessary to keep the gray level of the whole cleaned image on the same level which original image has. The result of this subtraction is presented in the Figure 3c. By this stage, the image contains only relevant information and is ready for further processing. 

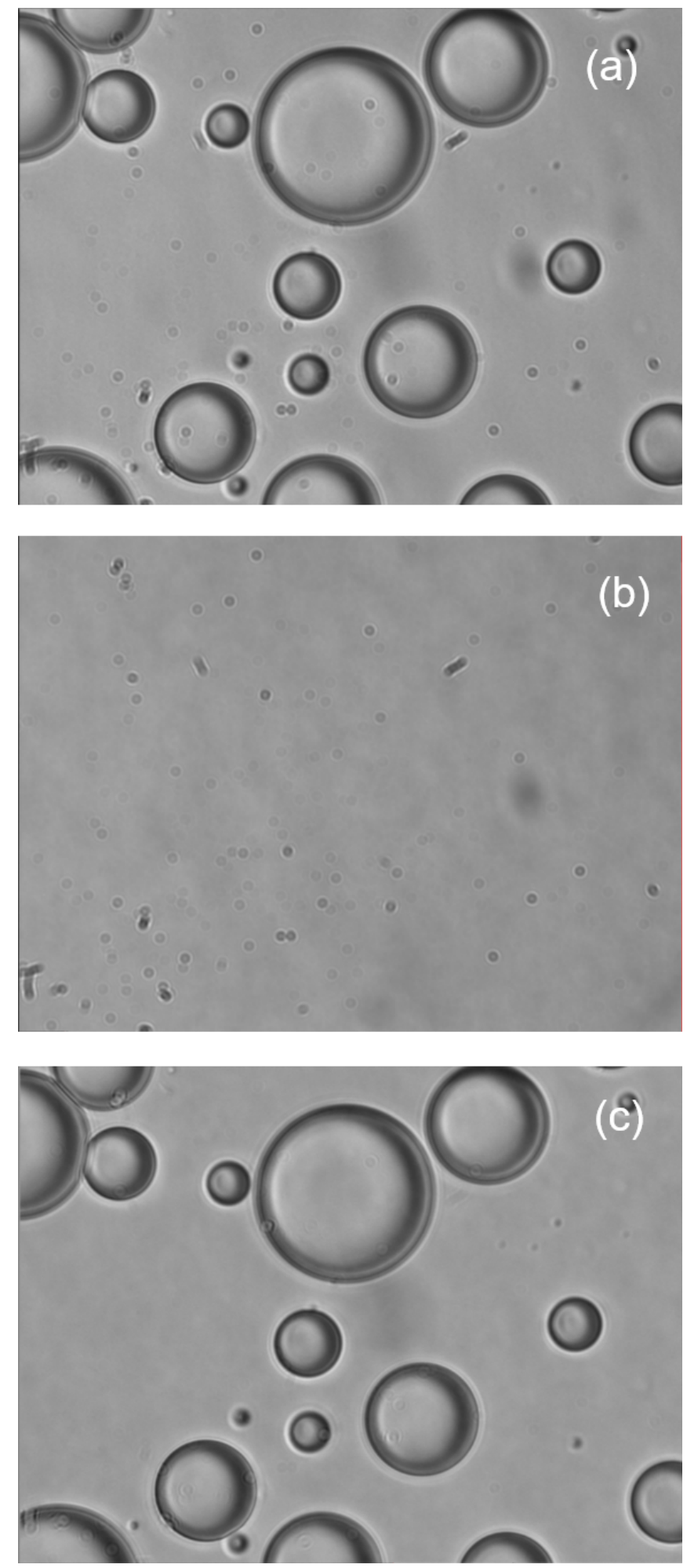

Figure 3. Explanation of Lens Cleaning method: a) original "dirty" image; b) extracted redundant information; c) "clean" image created by subtraction of a) and b). 


\subsubsection{Self quotient image (SQI)}

As mentioned earlier, not only the unnecessary objects visible in the image can be problematic for image analysis algorithm but also illumination variations may have a big impact on automatic detection. These cause nonuniform gray level in the image and all global actions such as thresholding become difficult to apply for one set of parameters. This problem can be overcome by Self Quotient Image (SQI) (Shashua \& Riklin-Raviv, 2001), an example of its usage are presented in (Gopalan \& Jacobs, 2010). Generally, SQI is applied to noisy images in order to increase the robustness of the automatic objects detection. It is achieved by normalizing intensity of every pixel based on its neighborhood. Name of this method is motivated by scheme of the calculations which are explained by Equation 3 (Maaß et al., 2012):

$$
Q(x, y)=\frac{B_{i}^{\prime}(x, y)}{X(x, y)}
$$

The normalized SQI image is created by the division of the original image by its blurred version (see Figure $4 \mathrm{a}$ and $4 \mathrm{~b}$ ). The smoothed image is then the result of the convolution between the original image and two-dimensional Gaussian kernel $(K)$. The size of this kernel determines the intensity of the the smoothing operation which, in turn, allows useful manipulation of the SQI images. The Equation 4 (Maa $\beta$ et al., 2012) below describes the application of this kernel.

$$
X=B * K
$$

This feature is truly important in the process for further analysis because it establishes the most appropriate SQI image. The two-dimensional Gaussian kernel size is available for the user through the first track-bar (Intensity of Blurring) showed in Figure 2. The image obtained is shown in the Figure 4c, where the main advantage of the operation is that the illumination variations have been removed and the histogram of the gray color is less spread. Moreover, borders of droplets are preserved and no important information has been lost.

\subsection{Image Analysis}

In most of the cases for droplet detection, conventionally, Hough transform has been employed; one of the optimized version of this algorithm is proposed by (Yuen, Princen, Illingworth, \& Kittler, 1990). The authors suggested that combination of the Gerig Hough Transform with a gradient (GHTG) and two stage Hough transform (2HT) is much more efficient and accurate than the traditional approach. This finding was so successful that it has been implemented to OpenCV library. Recently (Bras et al., 2009) proposed a variation of the Hough transform dedicated for automatic measurement of DSD for LLD. The reduced voting procedure which used only two sections of the right circular cone was applied. However, the sensitivity to noise and poor quality of images increased. Additionally, the computational costs, despite improvements, still stayed on a relatively high levels.

To overcome such issues, a new round objects detection procedure is presented which is not connected with already highly exploited Hough transform algorithm. Essentially 

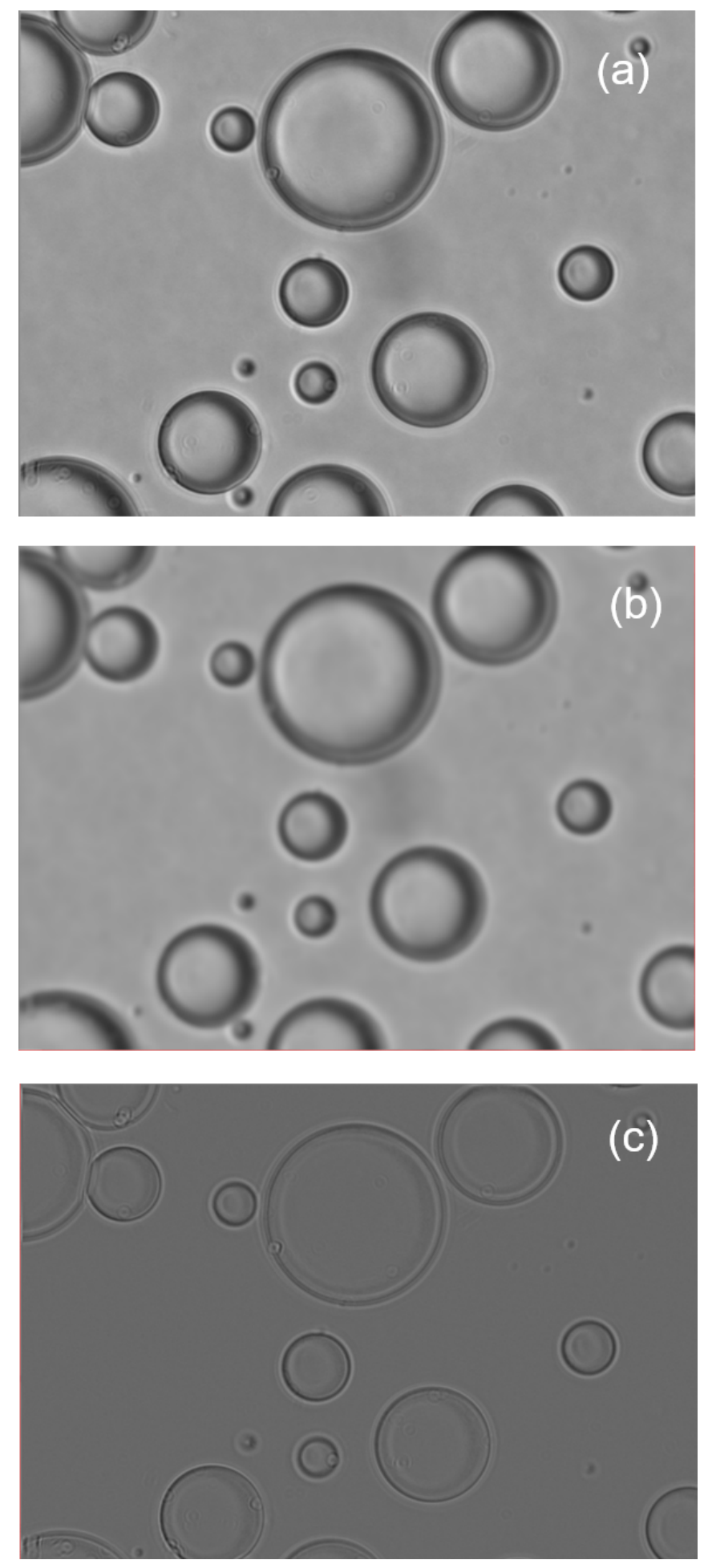

Figure 4. SQI method explanation: a) original "clean" image; b) blurred version of a); c) SQI image created by dividing a) by b). 
image analysis algorithm has been created in two steps. Firstly, the method of finding contours in an image is applied and secondly all detected contours are enclosed in circles whose sizes are as small as possible. After these two steps, next action is required and is described in Section 2.4 later.

\subsubsection{Finding contours}

In this step, the thresholded image of the SQI is a base for calculations. Borders existing in the image created by this operation now can be detected by the function "findcontours" implemented in the OpenCV library. This algorithm retrieves contours from the binary images as explained in (Suzuki, 1985). In their work, two algorithms were presented, where the first one determined the surroundness relations among the borders of a binary image and the second one followed only the outermost borders. Both algorithms can be effectively used for border detection however in this work only the second one is used. As the function "findcontours" requires a binary image, this is created by thresholding operation. This is when the second parameter (Brightness level) is manipulated through the middle track-bar as shown in the Figure 2. The Equation 5 below explains how the brightness parametrization works. During division of the original image by its blurred version the final result is multiplied by the value selected by the user.

$$
Q(x, y)=\text { multiplying_factor } * \frac{B_{i}^{\prime}(x, y)}{X(x, y)}
$$

The interval of the selectable values of the multiplying factor has been settled between 95 and 115 (based on experience). This operation has an influence on the final binary image as the thresholding operation is based on the constant value of the threshold. This has been established experimentally during the development stage and its value is set to 97 points of the gray color intensity. Thresholded image created by combination of the brightness manipulation and thresholding is shown in the Figure 5 a.

In the Figure $5 \mathrm{~b}$ detected contours are presented. As shown, the strongest contours are on the borders of droplets however, many other contours have also been identified. This is due to the "findcontours" function which is not concerned with the specific shape of an object (for example the round objects which is the main aim for the Hough transform). Thus, it looks for all contours presented in the image. This procedure is computationally less demanding which is a huge advantage however, further processes is required to achieve the desired results which in our case is the measurement of DSD using correct automatic droplets detection.

\subsubsection{Find minimal enclosing circles}

In this step another OpenCV function "minEnclosingCircle" is utilized. This function finds the minimal enclosing circle of a two-dimensional point set using an iterative algorithm by (Welzl, 1991). The function obtains information about the radius and the center of each circle and the data thus obtained is relevant for further processing which is performed in the further steps of the algorithm. The result of the detection of minimal enclosing circles is presented in Figure 6a. 

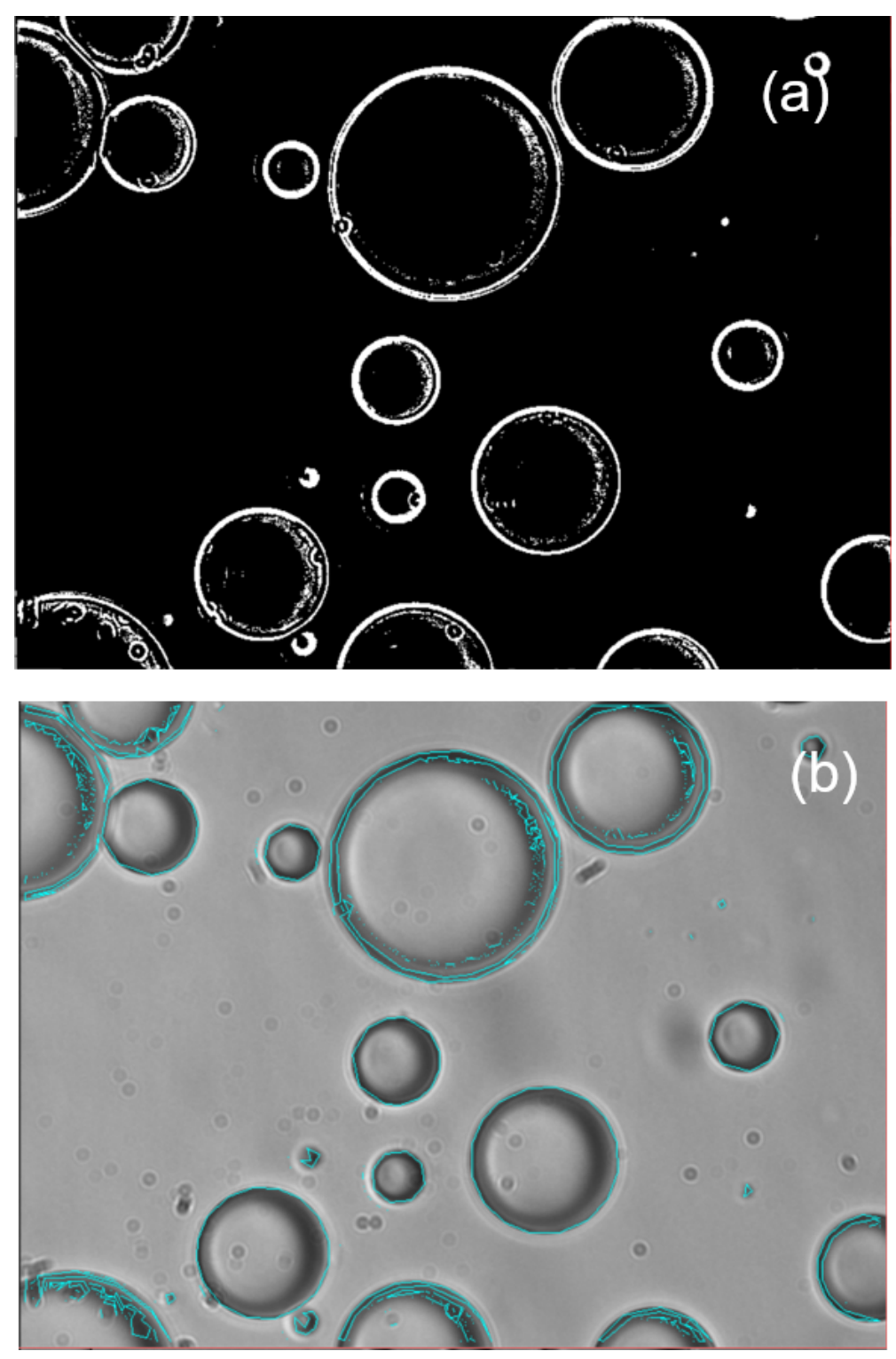

Figure 5. Result obtained by the "findcontours" OpenCV function: a)binary image analysed by algorithm; b) detected contours 


\subsection{Results Verification}

The combination of two described functions available in OpenCV library is an effective method for contours detection but not for detection of round objects itself. It makes calculations faster and easier, however together with simplicity, which is undeniably advantageous, enables the significant reduction of image analysis time in comparison to Hough transform. Further improvements are required in order to increase the accuracy which at this stage is very low. This is carried out in three steps as below.

\subsubsection{Delete small circles}

After detection of minimal enclosing circles most of the detections are circles with a radius equal or even smaller than 5 pixels. Such small droplets in dispersions are very rare but in a case when they truly exist it is almost impossible for correct detection even in a manual procedure. Therefore, initially the verification step deletes all circles where radii are equal or smaller than 5 pixels. It means that designed algorithm is unable to detect such small droplets but on other hands, it does not have a significant influence on the accuracy of the final results. The results obtained after deleting all small circles which met the condition is presented in the Figure 6b:

$$
\text { if(radius } \leq 5) \Longrightarrow \text { delete_circle }
$$

\subsubsection{Delete circles inside}

Despite achieving improvements after the first step of the verification process, usually a significant part of the circles is still wrong. Mainly it happens because of the shadows inside droplets which during image analysis for the find contours process are treated as an independent part, not connected with correct droplet border. In most of the cases, it leads to the appearance of small droplets (but big enough to pass first verification step) placed inside bigger droplet, usually a correctly detected circle. To remove these erroneous circles, a new verification process is needed which is able to find out a relation between existing circles. It can be done by using simple Cartesian plain geometry and comparison process enabling to compare circles to one another in terms of their location and sizes. The gray image is a two-dimensional space containing a number of points equal to multiplying operation of its height and width, rendering well-known equation for calculation of the distance between two points is valid here. An example of a possible situation when one of the circle is placed inside other is explained through the Figure 7 and Figure 8.

In the Figure 8 the rule of work of the comparison algorithm is presented. Every single circle is compared to other circles as it is shown on an example of the circle 5 in the Figure 7. During this comparison, the algorithm makes decisions whether the circle is correct or not and the result of this decision process is shown as a middle vector called "verification result" which is colour-coded (green colour means that circle is correct and the red colour means that circle is wrong). As a result of this verification process, a new vector is created which is called the "final vector". This vector contains only correct circles and is ready for further processing.

The procedure is based on circle center locations and radii sizes. Images in OpenCV library have their origins always in the upper left corner. For implemented algorithm, the unit of measurement is a pixel. During creation of minimal enclosing circles the "minenclosedcircle" function delivers information about the radii and centers in a form of two separated vectors. This knowledge is relevant and is used in two steps where 

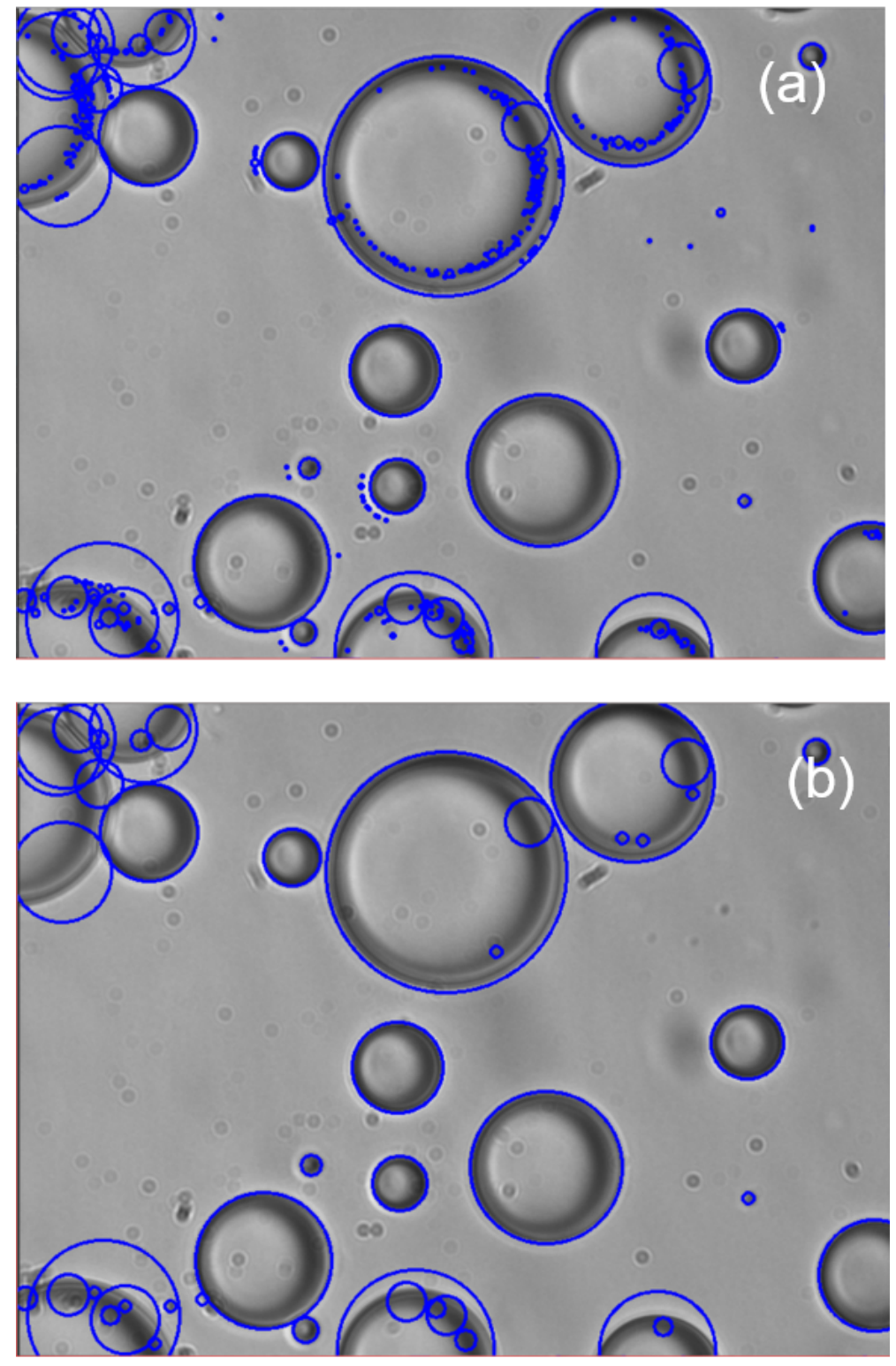

Figure 6. First verification step - deletion of small circles: a) image before verification; b) image after verification 


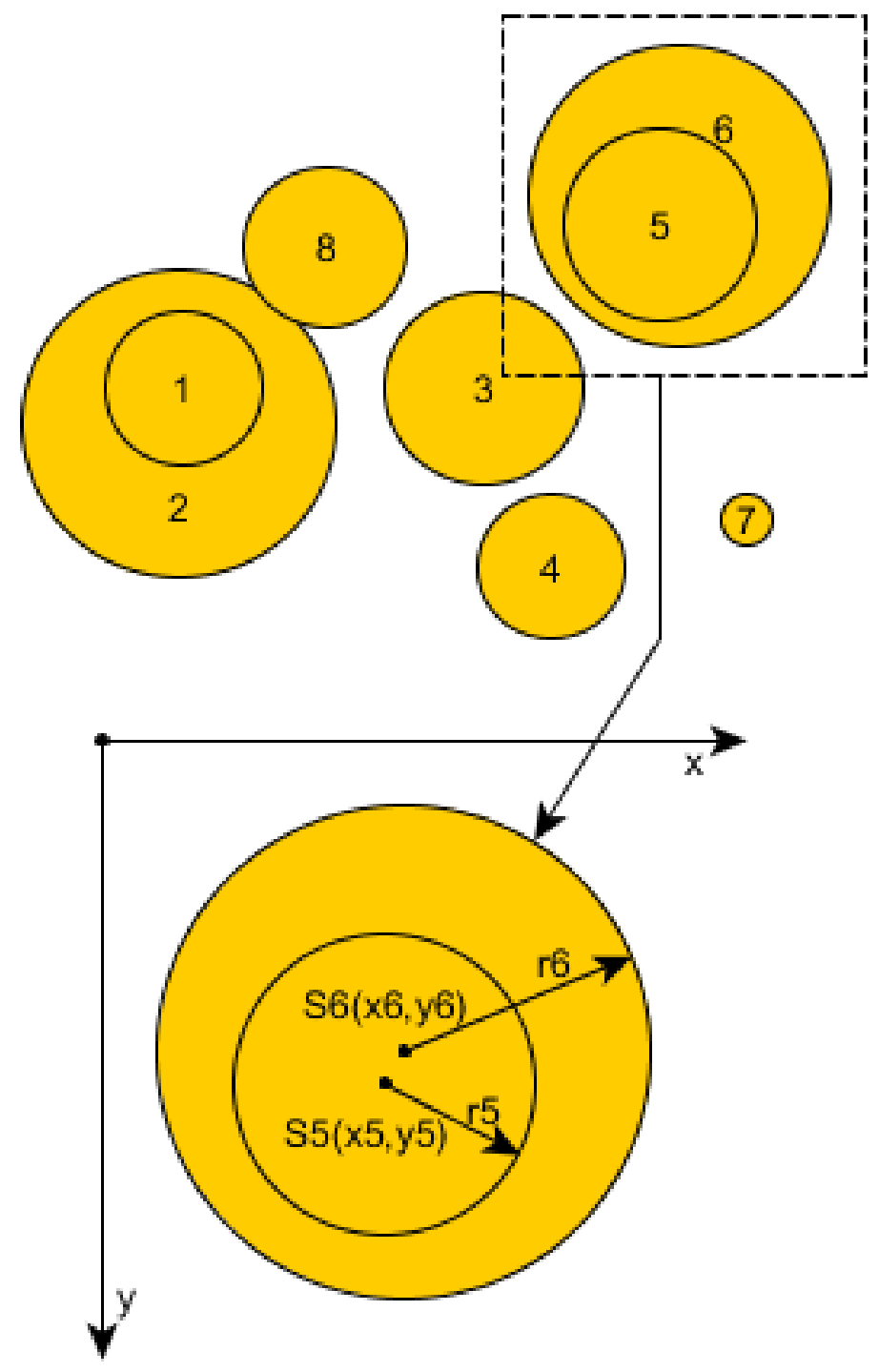

Figure 7. An example of the possible situation when one droplet is placed inside other one. 


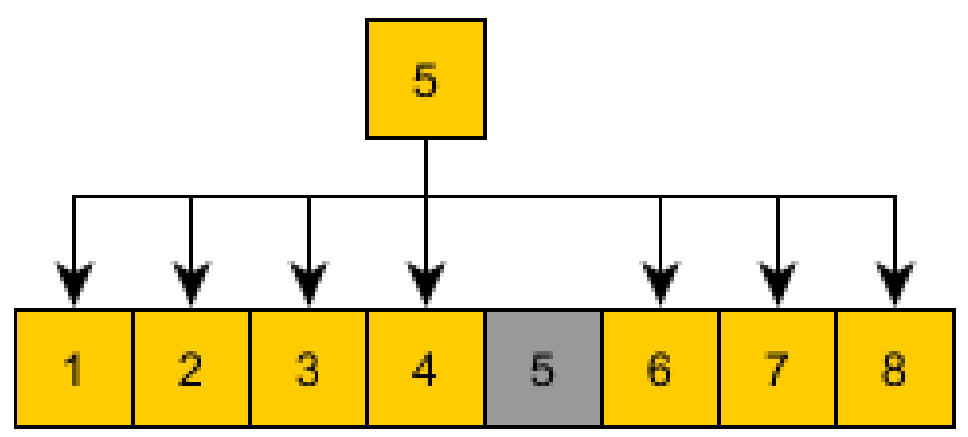

unverified vector

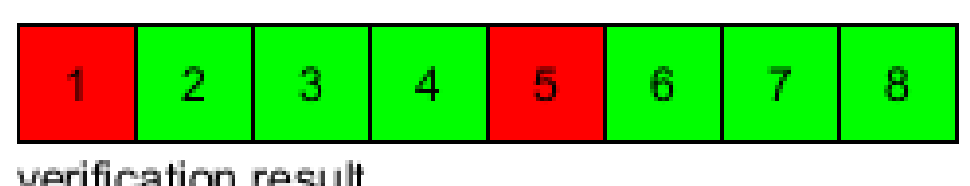

verification result

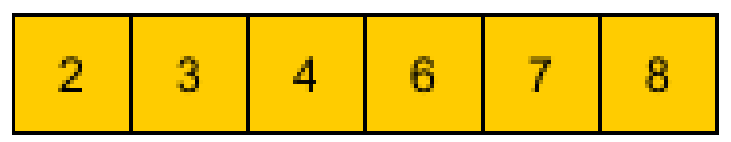

\section{final vector}

Figure 8. Testing procedure of the second verification step.

each of them checks different condition. In the first step, the distance between two centers is checked. In a case of highlighted droplets in Figure 7, the location of the center $\_5$ is denoted as $S 5(x 5, y 5)$ and for centre 66 as $S 6(x 6, y 6)$. The Equation 6 below states how the distance between these points is calculated.

$$
\text { distance }_{5-6}=\sqrt{\left(S x_{6}-S x_{5}\right)^{2}+\left(S y_{6}-S y_{5}\right)^{2}}
$$

After this, a length of the radius_5 is compared with calculated distance and in a case when it occurs to be shorter first condition is satisfied and the second condition is checked. The first condition can be written in following way:

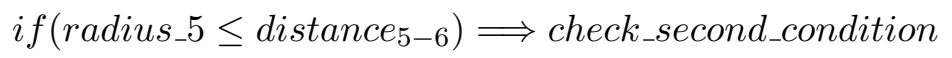

The second condition is needed as satisfying the first condition only means that one of the circle is inside the other but still it is impossible to determine which one should be deleted. This problem is solved by comparing the lengths of the radius_5 and radius_6. Based on experience from industry, it is more likely that a circle with a smaller radius is wrong and should be deleted. Thus, the second condition can be written as:

$$
\text { if }(\text { radius_5 } \leq \text { radius_6 }) \Longrightarrow \text { delete_circle_5 }
$$

In Figure 9b, the results of the second verification are presented. Now almost every wrong circle has been deleted and accuracy is higher. As a comment, it is important to say that this method is effective but its drawback is that the droplets detection 
algorithm is unable to detect droplets when more than half of their surfaces overlap. Moreover, some more imperfections can still be removed but for this improvement the third and last verification is required.

\subsubsection{Delete wrong circles}

This step can be referred to as the border verification because the base for it is the most important feature due to which droplets are visible on acquired images. Looking at the pictures of the emulsion it is clearly visible that despite several differences between droplets taken from various types of the emulsion, one feature is constant and common to all of them, i.e., the dark borders. This fact was noticed, for example, by (Lin, Skaf, \& Jennions, 1990) which used the gradients at the edges of droplets for the development of their image analysis approach. This phenomenon occurs due to the distortion of light caused by changes in density of the environment in which it is dispersed. Before the last verification can be applied it is required to create an image with extracted borders of droplets. Here, the last user interference is needed and the bottom track-bar (presented in Figure 2) enables it. The track-bar changes a value of the threshold which helps extract borders of droplets to the binary image. For this purpose the image created during lens cleaning processing is taken as a base for the thresholded image. The result of the operation is shown in the Figure 10.

As it was mentioned after second verification step, the accuracy of the results obtained is increased but a few wrong droplets can still be observed, especially on the borders of the image. Circles indeed are drawn because in these areas the droplets exist but the precision is away from the ideal. It happens due to the fact that during image acquisition these droplets have their centers beyond the scope of camera objective. As the specification of the automatic droplets detection is concentrated on enclosing contours in circles, for correct measurement at least half of the droplet surface is required. However, not only partially visible droplets are problematic but also non-circular objects with gray colour intensity (which is similar to droplet edges) can cause wrong detections. To overcome this disadvantage new methodology has been presented and the edges of the droplets are the most important feature helping verify whether detected circle truly represents a droplet or not. Figure 11 presents the main idea of the method. Eight square points placed inside detected circle check values of the pixels which are in their scope. Scope of each checkpoint depends on size of the detected circle. The condition that determines the size of the checkpoints is as follows:

$$
\text { if }(\text { radius }<40[\text { pixels }]) \Longrightarrow \text { size }=3[\text { pixels }] \text { else } \Longrightarrow \text { size }=0,1 * \text { radius }[\text { pixels }]
$$

The values of checking pixels are taken from the binary image (Figure 10b). The dynamic size of the checkpoints is applied to protect from inappropriate deletions of the big droplets which, due to the intensive light distortion on their borders, may be over estimated. This slight imprecision does not have an impact on the accuracy of the overall measurement but may cause unnecessary removal of correctly detected droplets. For the correct verification process, it is crucial to appropriately allocate the checkpoint. The main requirement is that the checkpoints always check pixels inside of the detected circle. It is possible by correct evaluation of the position of each checkpoint. In the code, it has been realized by referring to the center location of the circle. Next, for each of the square checkpoint bottom left vertex coordinates are determined and then direction of the checking procedure is determined according to the rule that it should converge to the center of the circle:

checkpoint_1: 

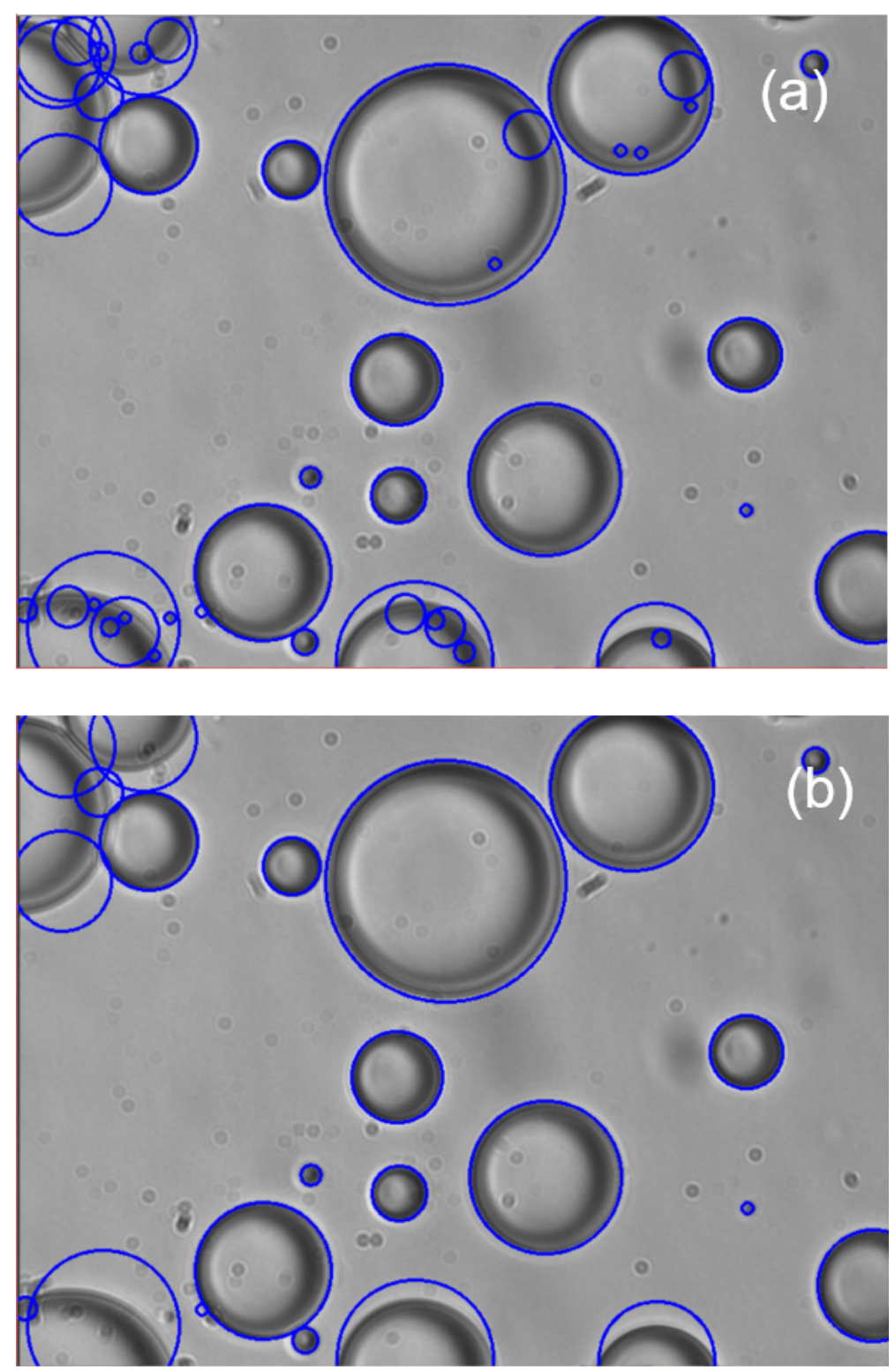

Figure 9. Second verification step - deletion of the circles placed inside other ones: a) image before verification; b) image after verification. 

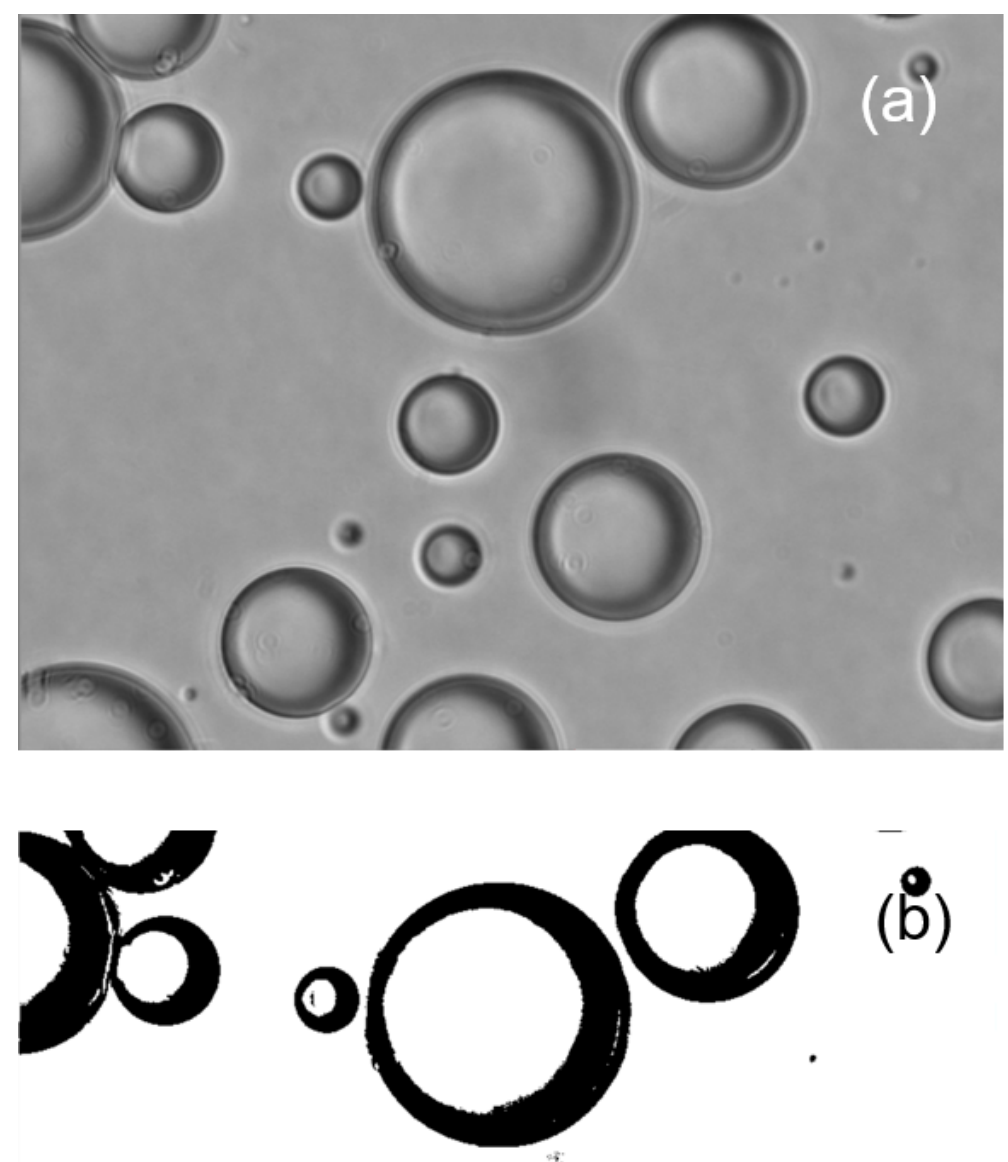

(b)

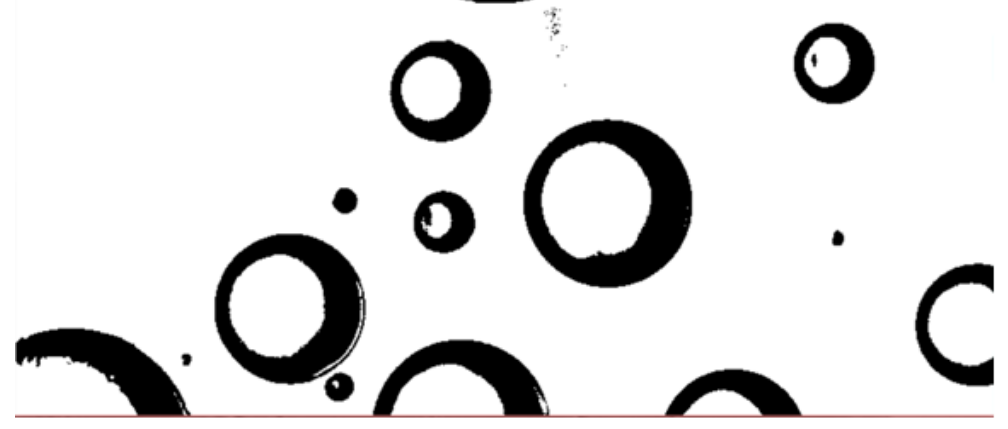

Figure 10. Thresholding operation for the third verification step: a) original "clean" image; b) thresholded version of a). 


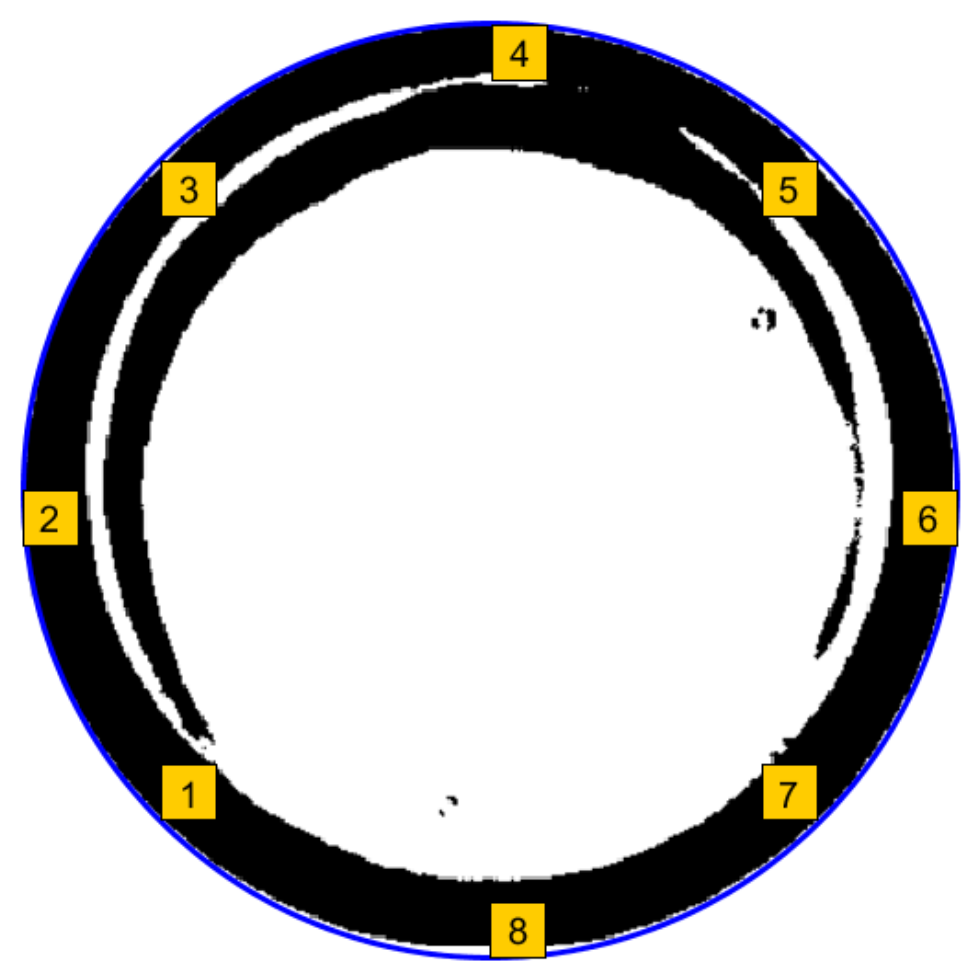

Figure 11. Locations of the checkpoints. Blue ring represents detected circle; Black ring is a thresholded version of the droplet.

$$
1_{x}=S_{x}-\frac{(\text { radius } * \sqrt{2})}{2} ; 1_{y}=S_{y}+\frac{(\operatorname{radius} * \sqrt{2})}{2}
$$

direction $_{x}=1_{x}+$ size $;$ direction $_{y}=1_{y}-$ size

checkpoint_2:

$$
2_{x}=S_{x}-\text { radius } ; 2_{y}=S_{y}
$$

direction $_{x}=2_{x}+$ size $;$ direction $_{y}=2_{y}+$ size

checkpoint_3:

$$
\begin{gathered}
3_{x}=S_{x}-\frac{(\text { radius } * \sqrt{2})}{2} ; 3_{y}=S_{y}-\frac{(\text { radius } * \sqrt{2})}{2} \\
\text { direction }_{x}=3_{x}+\text { size } ; \text { direction }_{y}=3_{y}+\text { size }
\end{gathered}
$$

checkpoint_4:

$$
4_{x}=S_{x} ; 4_{y}=S_{y}-\text { radius }
$$

direction $_{x}=4_{x}+$ size $_{\text {irection }}=4_{y}+$ size

checkpoint_5:

$$
\begin{gathered}
5_{x}=S_{x}+\frac{(\text { radius } * \sqrt{2})}{2} ; 5_{y}=S_{y}-\frac{(\text { radius } * \sqrt{2})}{2} \\
\text { direction }_{x}=5_{x}-\text { size }_{\text {rirection }}=5_{y}+\text { size }_{\text {ire }}
\end{gathered}
$$

checkpoint_6: 


$$
\begin{aligned}
& 6_{x}=S_{x}+\text { radius } ; 6_{y}=S_{y} \\
& \text { direction }_{x}=6_{x}-\text { size } ; \text { direction }_{y}=6_{y}+\text { size }
\end{aligned}
$$

checkpoint_7:

$$
\begin{gathered}
7_{x}=S_{x}+\frac{(\text { radius } * \sqrt{2})}{2} ; 7_{y}=S_{y}+\frac{(\text { radius } * \sqrt{2})}{2} \\
\text { direction }_{x}=7_{x}-\text { size } ; \text { direction } y=7_{y}-\text { size }
\end{gathered}
$$

checkpoint_8:

$$
\begin{gathered}
8_{x}=S_{x} ; 8_{y}=S_{y}+\text { radius } \\
\text { direction }_{x}=8_{x}+\text { size } ; \text { direction }_{y}=8_{y}-\text { size }
\end{gathered}
$$

Obviously, the number of checking pixels depends on the size of the checkpoint and is equal to size $e^{2}$. Checkpoint is marked as correct if at least one pixel inside its scope is black. The black pixel means that checkpoint is placed on the edge of droplet. When all checkpoints have their value, number of checkpoints marked as wrong is calculating and following condition is inspected:

$$
\text { if (number_of_wrong_checkpoints } \geq 2) \Longrightarrow \text { delete_circle }
$$

The condition allows for existing one wrong checkpoint and all test which will be presented in next chapter has been evaluated according to this exception. Changing this parameter is not available to the user however examination of available samples proved that the permission for one wrong checkpoint is the most optimal. For the condition where no wrong checkpoints are allowed and where two wrong checkpoints are allowed, it resulted in respectively $28,4 \%$ less and $13,8 \%$ more droplets detected than for the semi-automatic detection which is treated as the most accurate method for counting droplets. The final results look well after three verification procedures, as in the Figure 12b. All droplets on the edges of the image that are beyond the viewing range are ignored, and only properly detected droplets remained in the image.

\section{Results}

\subsection{Testing Procedure}

Several experiments were conducted to evaluate the reliability of the designed algorithm for automatic droplet detection, its robustness to changes in the droplet concentration, image quality and many other image properties which always change during image acquisition of different LLD systems. Various batches of images were used which were provided by an industrial collaborator which assures that the algorithm is based on true data useful for industry. Moreover, each image is different in various aspects (from density of droplets to the image quality). It provides good opportunity to carry out validation procedure for code development on a variety of test cases. In order to perform verification objectively, normalized work-flow has been defined in the Figure 13 which helps achieve results which can be easily compared with each other. The assessment scheme is divided into three possible ways. For all batches of images three variants of automatic, semi-automatic and manual approach has been checked. A temporal assessment has been carried out for the manual approach for the DSD measurement. For comparison of efficiency of the automatic droplets detection, the 

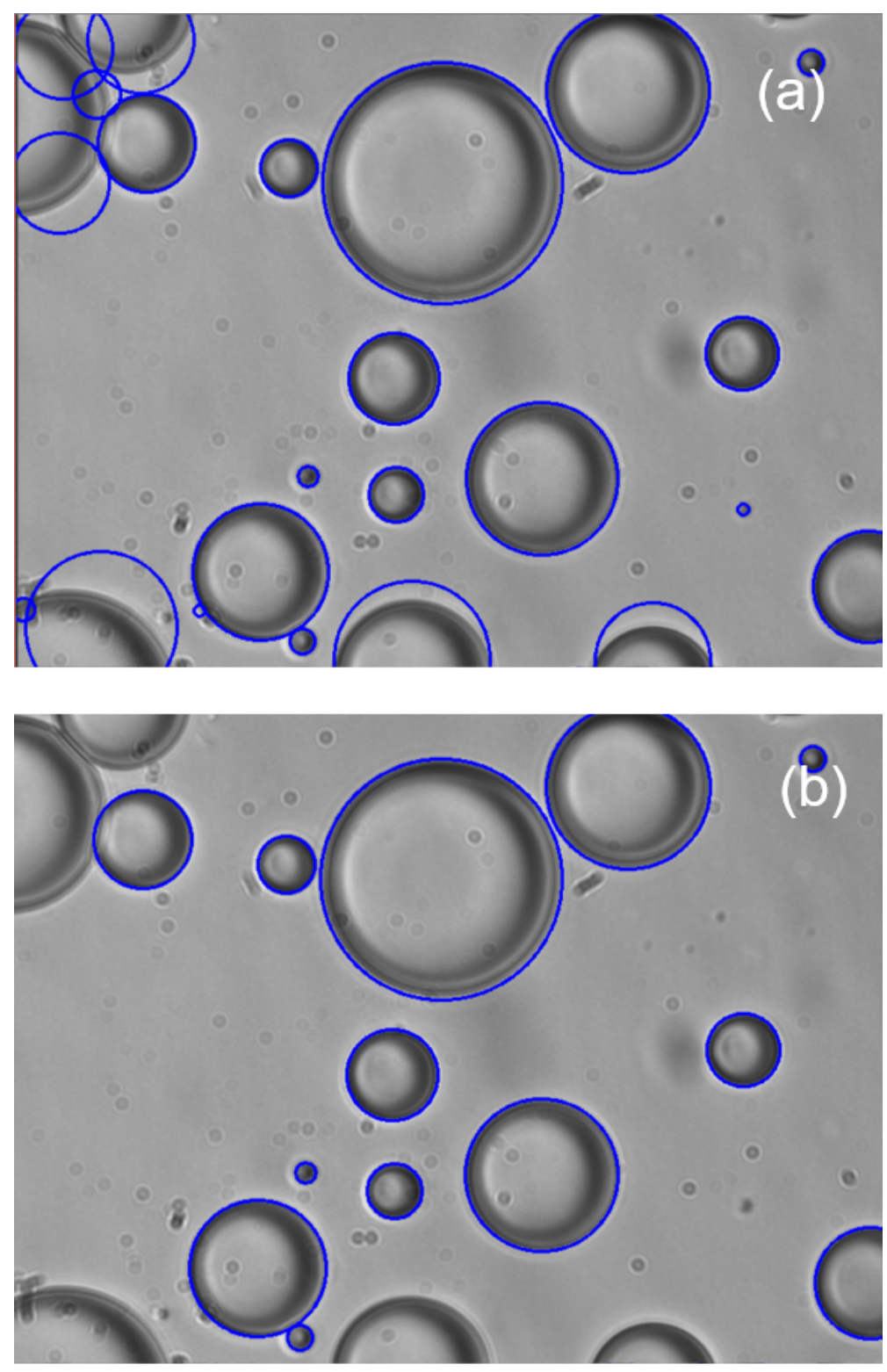

Figure 12. Third verification step - deletion of the wrongly recognised circles: a) image after verification; b) image after verification. 


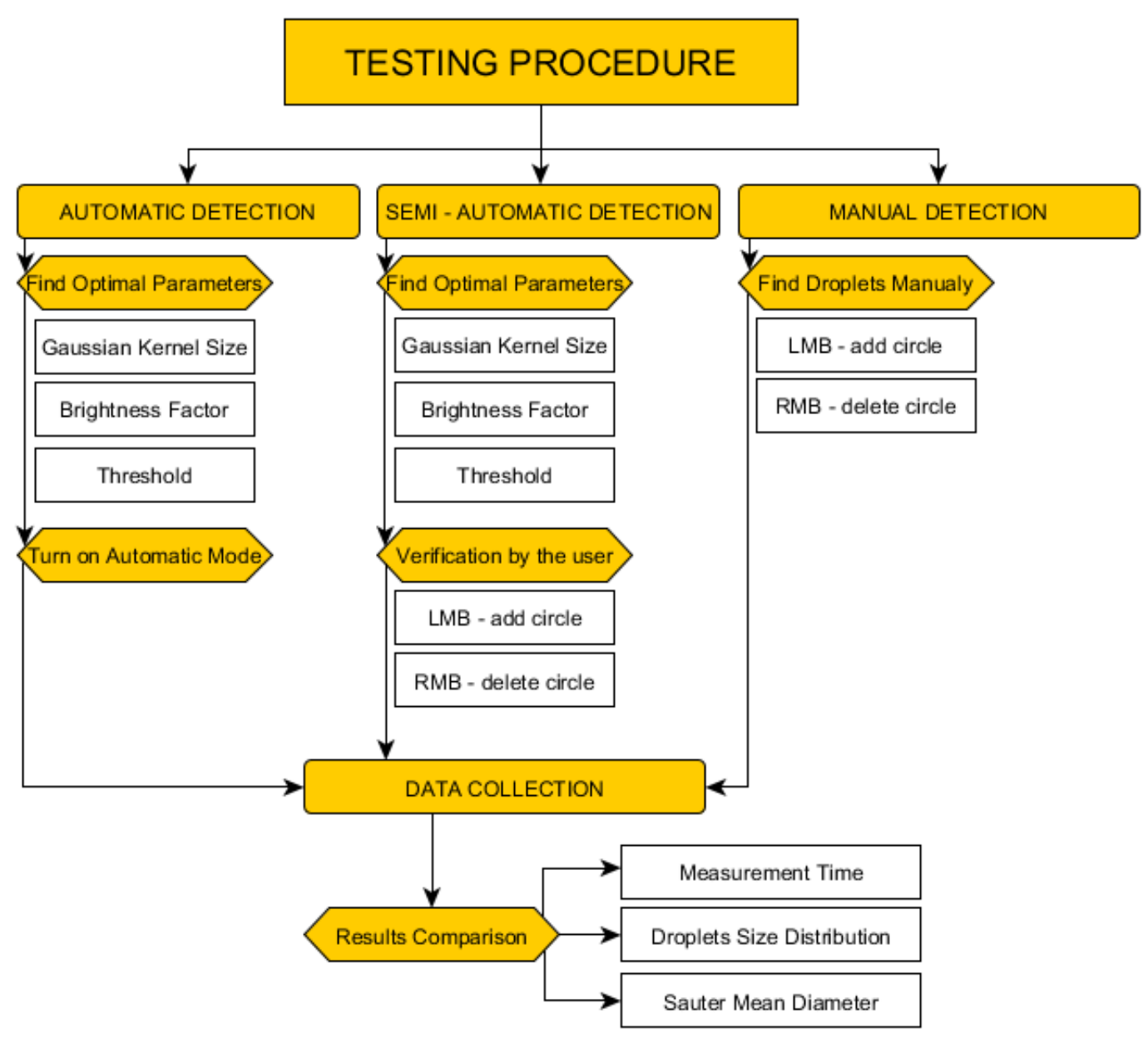

Figure 13. Work-flow of the testing procedure.

semi-automatic detection is considered as the most accurate method where all detections are correct. Therefore, the results of the automatic detection are compared to the results of the semi-automatic detection.

\subsubsection{Automatic droplets detection}

The first step before activation of the automatic mode is a parametrization of the three factors which were discussed in the earlier section. It means that automatic detection is not truly automatic detection because it still requires some user attention. However this drawback exists only at the beginning of the automatic mode and after relevant adjustments the algorithm is ready for analysis on the given batch of sample images. Usually, after first two or three images, it is possible to determine the most optimal values of these factors for whole batch. It warranties that the algorithm can cope with automatic measurement without any problems even totally new type of images is tested. In the other words, in exchange for a small workload associated with parametrization, the user receives a universal computer program. 


\subsubsection{Semi-automatic droplets detection}

The first step of the semi automatic mode is same as for the automatic mode. The user has to decide which set of parameter is the most optimal for analyzed sample of images. However, after this step, further verification is still required; this verifications process is carried out by manually adding miss-detected droplets on the image. Similarly, when the wrong circle was detected during automatic analysis it can be removed manually. In our opinion, this approach is the fastest and the easiest. However, other possibility exists were the parameter adjustment for every single image is performed. This makes sense when most of the droplets were not detected for the previous set of parameters and manual addition/removal could be more time consuming than establishing a new set of parameters.

\subsubsection{Manual droplets detection}

Manual droplets detection is independent mode and all measurements are made by the user. It allows to obtain estimated time required for manual DSD measurement which can be compared with the time acquired during the automatic and the semi-automatic measurements.

\subsubsection{Data collection}

As the last step of testing procedure common to all modes is data collection were data is output in ASCII format and contains all captured information such as the number of detected droplets, diameters of detected droplets and time required to carry out the measurements. All these are required for further analysis to understand the performance of the algorithm. In this case, the results obtained have been examined in three important aspects determining usability of the designed code. The most important one is the measurement of DSD which is the main factor describing emulsion, second is Sauter mean diameter and its variations which are strongly connected with the DSD and defines emulsion as one droplet diameter. The last one is the time it takes for the measurements which is helpful in understanding the economical performance of the algorithm.

\subsection{Droplets Size Distribution}

In this section results in terms of number of detected droplets are presented. For clear understanding, droplets are segregated according to their sizes. It creates a histogram and shows how sizes of the droplets dispersed in the emulsion, are spread. The comparison between automatic and semi-automatic approach is presented graphically using charts, which provide information regarding the sizes of the droplets for which the automatic approach faced challenges. Additionally, based on an overall number of detected droplets it is possible to calculate the percentage droplets in each range of the diameters which is the fundamental aim of the measurement of DSD. It must be mentioned that if the bars representing automatic and semi-automatic detections are not equal, it does not mean that the final result are incorrect; more important aspect is to keep the same distribution of the droplets sizes. In the other words, dotted lines which are added to every chart should be as close as possible to each other. Only this feature has impact on the emulsion's description. Characteristic features of the images (examples are shown in Figure 14) which were used for algorithm evaluation are: 
- High and medium amount of the dispersed phase.

- Sizes of droplets are medium and small and the range is not very wide.

- Most of the droplets have similar contours which are dark and well visible.

- Droplets are close together but overlap phenomenon does not occur often.

- Clean images with good quality and uniform gray level.

Images in this sample are challenging and complex due to high density of the dispersed phase. However, results in terms of number of detected droplets shown in Figure 15 is satisfying. Here, the most of the error occur in the smallest range of droplets sizes where automatic method found over 20 percent more droplets than the manual approach. This drawback is visible for almost every analysed batch of images. On the other hand, rest of the droplets are detected with a difference around 10 percent or less. This fact reduces the influence of wrongly detected smallest droplets on the calculation of the emulsion parameters such as Sauter Mean Diameter (results in Section $3.3)$.

\subsection{Sauter mean diameter}

Performing a droplets size analysis is the best way to answer the question: What size are those droplets? Once the analysis is complete the user has a variety of approaches for analyzing the result. One of them is the calculation of a mean diameter which delivers information about an average size of the droplets dispersed in the emulsion. This can partially explain specification of the mixture and determine further actions for the development of better emulsification processes.

The two types of mean diameter are considered as follows:

- Surface area moment mean D[3, 2] - so called Sauter Mean Diameter, is most relevant where specific surface area is important e.g. bioavailability, reactivity, dissolution. It is most sensitive to the presence of fine droplets in the size distribution

- Volume moment mean $\mathrm{D}[4,3]$ so called De Brouckere Mean Diameter, is relevant for many samples as it reflects the size of those particles which constitute the bulk of the sample volume. It is most sensitive to the presence of large droplets in the size distribution.

The Sauter mean diameter can be calculated as below:

$$
D[3,2]=\frac{\sum_{1}^{n} D_{i}^{3} v_{i}}{\sum_{1}^{n} D_{i}^{2} v_{i}}
$$

The base for this equation is a histogram chart which shows the upper and lower limits of n size channels (Figure 15). The $D_{i}$ value for each channel is the geometric mean, as shown in the Equation 8 below:

$$
D_{i}=\sqrt{\text { upper_bound } * \text { lower_bound }}
$$

In the Equation 7, the numerator takes the geometric $D_{i}$ to the third power multiplied by the percent in that channel, summed over all channels. For the denominator 

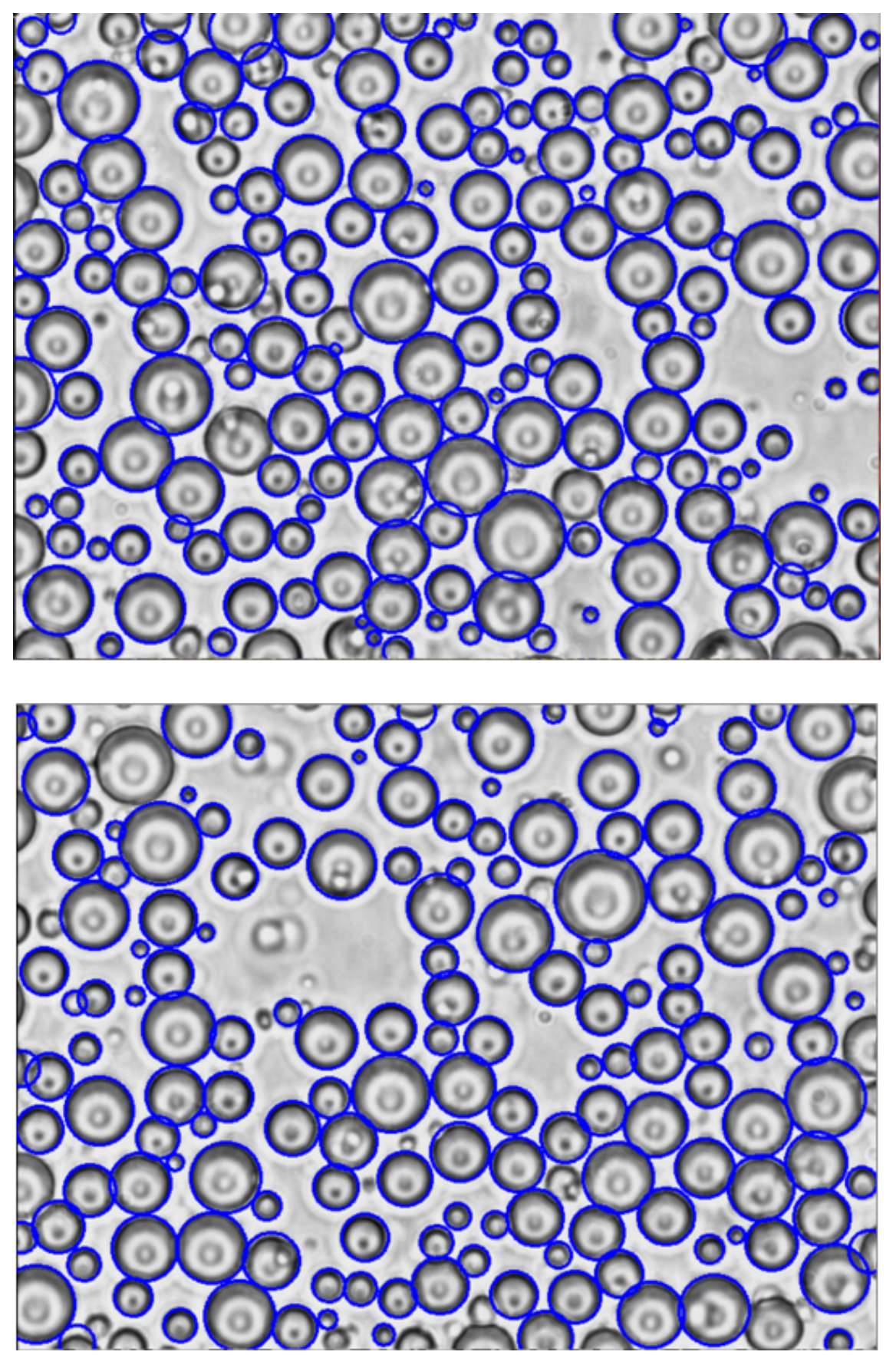

Figure 14. Example of analysed images which were used for evaluation of the novel automatic droplets detection algorithm. 


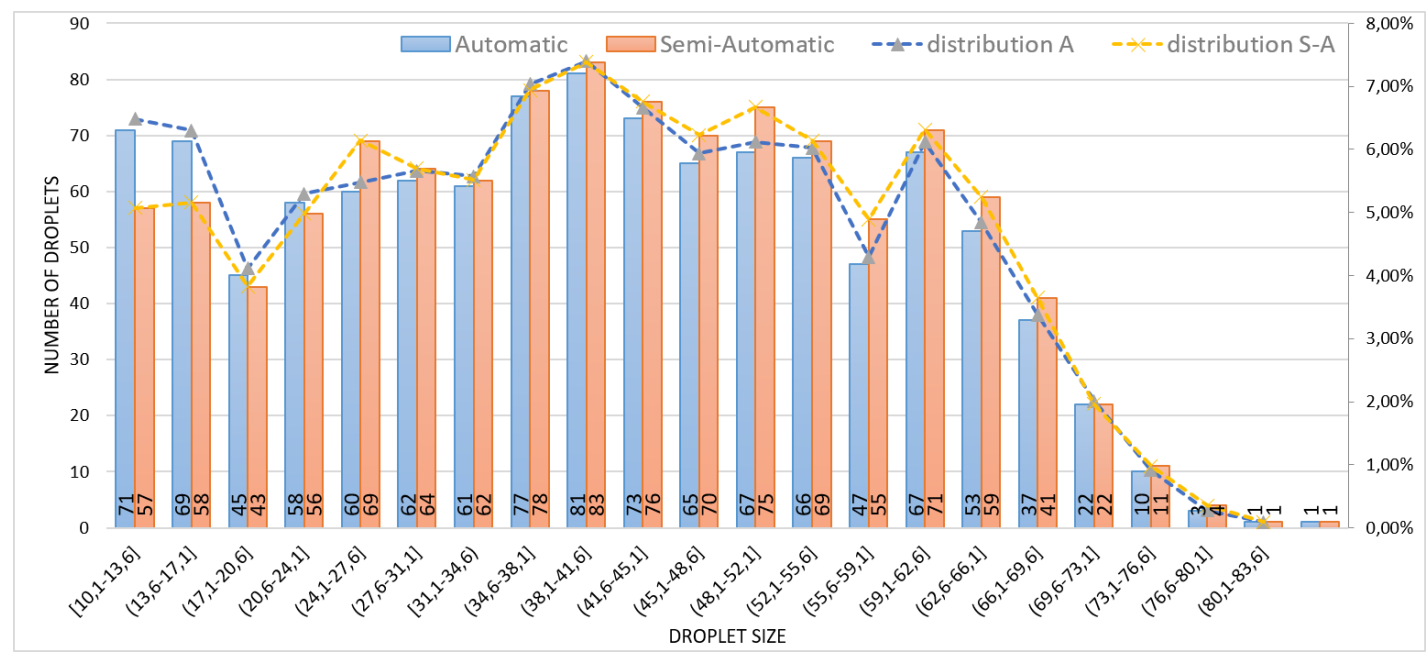

Figure 15. Number of detected droplets and droplet size distribution obtained by automatic and semiautomatic method. 7 analysed images; 1096 detected droplets (AUTO); 1125 detected droplets (SEMI-AUTO).

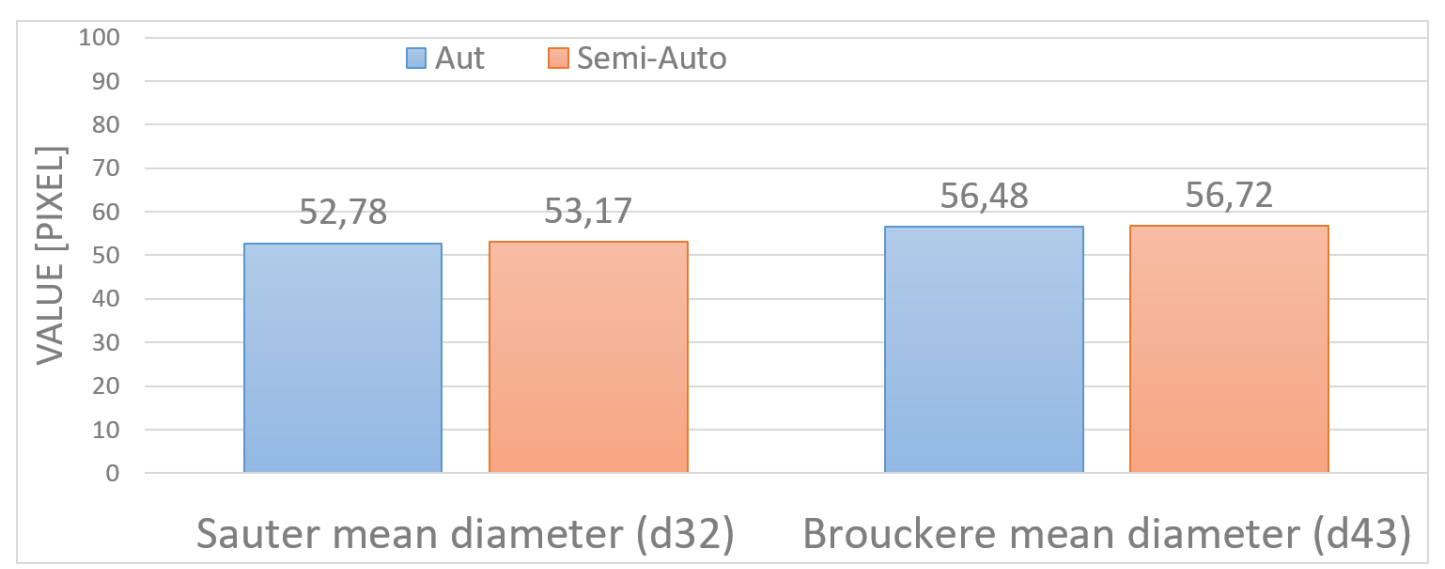

Figure 16. Comparison of the obtained mean diameter (type $d_{32}$ and $d_{43}$ ).

takes the geometric $D_{i}$ to the second power multiplied by the percent in that channel, summed over all channels. The procedure for the De Brouckere Mean Diameter is the same but instead of third and second power fourth and third powers are used respectively. Results of this calculations are presented in Figure 16.

\subsection{Measurement time}

The algorithm developed has also been tested in terms of its economical benefits in practical use. In the code, a timer is implemented for measuring the time when a batch of images is delivered to computer application and stops measurement when the last image in the batch has been analyzed. Time of the measurement has been check for three approaches: manual, semi-automatic and automatic. Figure 17 presents a comparison the three approaches in terms of time each method takes. The analysis shows that the automatic detection is almost 100 times faster than manual detection and almost 13 times faster than the semi-automatic. This is a huge benefit for using the proposed algorithm for the measurement of DSD in a industrial environment whether 


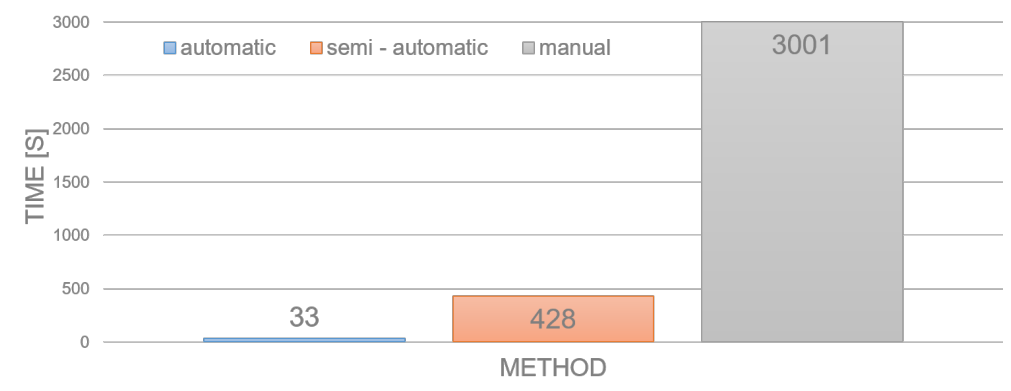

Figure 17. Time consumed for each droplets detection approach. Average number of droplets: 1100.

fully automatic or even semi automatic modes. Referring to the previous tests concerned with the accuracy of the measurement, developed code is not only precise in calculations but also offers huge improvements in terms of time.

\section{Conclusions and Future Work}

In this article, an automatic droplets detection method based on image processing and image analysis operations has been presented. The objective of this algorithm is the analysis of the images from an LLD process which (before the idea of this project) were analyzed in a traditional manual approach where analysis time was of major concern. Therefore, not only an accurate automatic droplets detection was required but also time reduction was the important part of the algorithm development. Extensive validation process shown in Section 3 demonstrates that the stated objectives are satisfied. Accuracy in most of the cases is close to $90 \%$ or better and the characteristic factors such Sauter mean diameter are calculated almost with the same precision as in method based on user inference. It leads to the conclusion that the novel method proposed in this report is able to detect a large proportion of the droplets.

Furthermore, various images were utilized in the testing process and it led to next conclusion that method is not only efficient but also able to deal with a variety of scenarios and features in the images. Mostly it has been achieved by implementation of algorithms such as contours detection and minimal enclosing circles recognition which are much less computational demanding than well known and widely used Hough transform. Proposed combination of these two methods along with the new techniques proposed here provides a promising approach which after further improvement may fully solve the problem of the on-line (in-situ) automatic droplets detection. However, some drawbacks of the method were observed after the testing and validation processes. Specifically, it pertains to false detections of the smallest droplets. This issue appears almost in every type of tested images. It is probably because of the noise and the characteristic feature of the methodology, which is very sensitive to this kind of aberrations. On the other hand, this issue does not leave negative influence on the Sauter mean diameter in any way.

Referring to the future work, the further validation process is needed to check how high density of dispersed phase make automatic detection problematic and impossible to overcome even with adjustment of the parameters provided for the user inference. Mentioning the parameter adjustment it is crucial to highlight that this is the only temporary solution and big effort should be devoted for development a way for automatic parametrization in future. In authors opinion, it is possible to obtain by finding 
the correlation between properties of the analyzed image such as average gray color value, an intensity of the borders, color histogram and the values of the most optimal set of parameters. Lastly, some research concerned on the comparison of the already exist programs in the industry with the method developed in this report could be done. It may give wider knowledge about achieved improvements by applying method differs to other methods mainly based on Hough transform and identify remains lack of understanding or a possible way of the further development.

\section{References}

\section{References}

Alban, F., Sajjadi, S., \& Yianneskis, M. (2004). Dynamic tracking of fast liquid-liquid dispersion processes with a real-time in-situ optical technique. Chemical Engineering Research and Design, 82(8), 1054-1060.

Arai, K., Konno, M., Matunaga, Y., \& Saito, S. (1977). Effect of dispersedphase viscosity on the maximum stable drop size for breakup in turbulent flow. Journal of Chemical Engineering of Japan, 10(4), 325-330.

Basaran, T., \& McClements, D. (1999). Nondestructive monitoring of sucrose diffusion in oil-in-water emulsions by ultrasonic velocity profiling. Journal of Colloid and Interface Science, 220(2), 429-435.

Bras, L. M. R., Gomes, E. F., Ribeiro, M. M. M., \& Guimares, M. M. L. (2009). Drop distribution determination in a liquid-liquid dispersion by image processing. International Journal of Chemical Engineering.

Chatzi, E., Boutris, C., \& Kiparissides, C. (1991). Online monitoring of drop size distributions in agitated vessels 1. effects of temperature and impeller speed. Industrial and Engineering Chemistry Research, 30(3), 536-543.

Chen, H., \& Middleman, S. (1967). Drop size distributions in stirred liquidliquid systems. AIChE J., 13(5), 989-998.

Cohen, M., \& Toussaint, G. T. (1977). On the detection of structures in noisy pictures. Pattern Recognition, 9(2), 95-98.

Coulaloglou, C., \& Tavlarides, L. (1976). Drop size distributions and coalescence frequencies of liquidliquid dispersions in flow vessels. AIChE J., 22(2), 289-297.

Crawley, G., \& Malcolmson, A. (2004). Online particle sizing as a route to process optimization. Chemical Engineering, 111(9), 37-41.

Galindo, E., Larralde-Corona, C. P., Brito, T., Crdova-Aguilar, M. S., Taboada, B., VegaAlvarado, L., \& Corkidi, G. (2005). Development of advanced image analysis techniques for the in situ characterization of multiphase dispersions occurring in bioreactors. Journal of Biotechnology, 116(3), 261-270.

Gopalan, R., \& Jacobs, D. (2010). Comparing and combining lighting insensitive approaches for face recognition. Computer Vision and Image Understanding, 114(1), 135145.

Junker, B. (2006, Aug 01). Measurement of bubble and pellet size distributions: past and current image analysis technology. Bioprocess and Biosystems Engineering, 29(3), 185206.

Kamp, J., Hnsch, R., Kendzierski, G., Kraume, M., \& Hellwich, O. (2016). Automated image analysis for trajectory determination of single drop collisions. Computers and Chemical Engineering, 89, 184-191.

Lagisetty, J., Das, P., \& Kumar, R. (1986). Breakage of viscous and non-newtonian drops in stirred dispersions. Chemical Engineering Science, 41(1), 65-72.

Lin, Y., Skaf, Z., \& Jennions, I. K. (1990). A bayesian approach to fault identification in the presence of multi-component degradation. Computers and Chemical Engineering, 14(11), $1201-1211$. 
Maaß, S., Rojahn, J., Hnsch, R., \& Kraume, M. (2012). Automated drop detection using image analysis for online particle size monitoring in multiphase systems. Computers and Chemical Engineering, 45, 27-37.

Maaß, S., Wollny, S., Voigt, A., \& Kraume, M. (2011). Experimental comparison of measurement techniques for drop size distributions in liquid/liquid dispersions. Experiments in Fluids, 50(2), 259-269.

Narsimhan, G., \& Goel, P. (2001). Drop coalescence during emulsion formation in a highpressure homogenizer for tetradecane-in-water emulsion stabilized by sodium dodecyl sulfate. Colloid and Interface Science, 238(2), 420-432.

Niknafs, N., Spyropoulos, F., \& Norton, I. T. (2011). Development of a new reflectance technique to investigate the mechanism of emulsification. Journal of Food Engineering, 104(4), 603-611.

Pacek, A., Moore, I., Nienow, A., \& Calabrese, R. (1994). Video technique for measuring dynamics of liquidliquid dispersion during phase inversion. Aiche Journal, 40(12), 19401949.

Ribeiro, M., Guimares, M., Madureira, C., \& Pinto, J. C. (2004). Non-invasive system and procedures for the characterization of liquidliquid dispersions. Chemical Engineering Journal, $97(2), 173-182$.

Richter, A., Voigt, T., \& Ripperger, S. (2007). Ultrasonic attenuation spectroscopy of emulsions with droplet sizes greater than $10 \mathrm{~lm}$. Journal of Colloid and Interface Science, 315(2), 482-492.

Ritter, J., \& Kraume, M. (2000). On-line measurement technique for drop size distributions in liquid/liquid systems at high dispersed phase fractions. Chemical Engineering and Technology, 579 .

Schalkoff, R. J. (1989). Digital image processing and computer vision. John Wiley and Sons, New York, NY, USA.

Shashua, A., \& Riklin-Raviv, T. (2001). The quotient image: Class-based re-rendering and recognition with varying illuminations. IEEE Transactions on Pattern Analysis and Machine Intelligence, 23(2), 129139.

Suzuki, S. (1985). Topological structural analysis of digitized binary images by border following. Computer Vision, Graphics and Image Processing, 30, 32-46.

Tcholakova, S., Denkov, N., \& Danner, T. (2004). Role of surfactant type and concentration for the mean drop size during emulsification in turbulent flow. Langmuir, 20(18), 74447458.

Tobin, T., Muralidhar, H., Wright, H., \& Ramkrishna, D. (1986). Determination of coalescence frequencies in liquidliquid dispersion: effect of drop size dependence. Chemical Engineering Science, 45, 3491-3504.

Welzl, E. (1991). Smallest enclosing disks (balls and ellipsoids). In New results and new trends in computer science: Graz, austria, june 20-21, 1991 proceedings (pp. 359-370). Berlin, Heidelberg: Springer Berlin Heidelberg.

Wright, H., \& Ramkrishna, D. (1994). Factors affecting coalescence frequency of droplets in a stirred liquidliquid dispersion. Aiche Journal, 40(5), 767-776.

Yan, S., Sayad, S., \& Balke, S. T. (2009). Image quality in image classification: Adaptive image quality modification with adaptive classification. Computers and Chemical Engineering, 33(2), 429-435.

Yuen, H., Princen, J., Illingworth, J., \& Kittler, J. (1990). Comparitive study of hough transform methods for circle finding. Image and vision computing, 8, 71-78.

Zhou, G., \& Kresta, S. (1998). Evolution of drop size distribution in liquidliquid dispersions for various impellers. Chemical Engineering Science, 53(11), 2099-2113.

Zhou, Y., Srinivasan, R., \& Lakshminarayanan, S. (2009). Critical evaluation of image processing approaches for real-time crystal size measurements. Computers and Chemical Engineering, 33(5), 1022-1035. 


\section{An automatic image analysis} methodology for the measurement of pÿdroplet size distributions in liquid liquid dispersion: round object detection

Gawryszewski, K.

ACTA Press

K Gawryszewski, ZA Rana, KW Jenkins, et al., An automatic image analysis methodology for pÿthe measurement of droplet size distributions in liquid liquid dispersion: round object detection. International Journal of Computers and Applications, Volume 41, Issue 5, 2019, pp. 329-342 https://doi.org/10.1080/1206212X.2018.1542555 Downloaded from Cranfield Library Services E-Repository 